\begin{tabular}{|l|l|}
\hline Title: & $\begin{array}{l}\text { Effects of coil configuration switching, pole-changing and multi-phase windings on } \\
\text { permanent magnet synchronous motors }\end{array}$ \\
\hline Authors: & Miriam Boxriker, Johannes Kolb, Martin Doppelbauer \\
\hline Institute: & $\begin{array}{l}\text { Karlsruhe Institute of Technology (KIT) } \\
\text { Elektrotechnisches Institut (ETI) } \\
\text { Hybrid Electric Vehicles (HEV) }\end{array}$ \\
\hline Type: & $\begin{array}{l}\text { Conference Proceedings } \\
\text { Published at: }\end{array}$ \\
$\begin{array}{l}\text { 43rd Annual Conference of the IEEE Industrial Electronics Society, IECON 2017, } \\
\text { Beijing, China } \\
\text { Publisher: IEEE, Piscataway (NJ) } \\
\text { Year: } 2017 \\
\text { ISBN: } 978-1-5386-1127-2 \\
\text { Pages: } 1925-1932\end{array}$ \\
\hline Hyperlinks: & DOI: https://doi.org/10.1109/IECON.2017.8216325 \\
\hline
\end{tabular}

(c) 2017 IEEE. Personal use of this material is permitted. Permission from IEEE must be obtained for all other uses, in any current or future media, including reprinting/republishing this material for advertising or promotional purposes, creating new collective works, for resale or redistribution to servers or lists, or reuse of any copyrighted component of this work in other works. 


\section{Effects of Coil Configuration Switching, Pole-Changing and Multi-Phase Windings on Permanent Magnet Synchronous Motors}

\author{
Miriam Boxriker \\ Elektrotechnisches Institut \\ Karlsruhe Institute of Technology \\ Karlsruhe, Germany \\ Email: miriam.boxriker@kit.edu
}

\author{
Johannes Kolb \\ SHARE at KIT \\ Schaeffler Technologies AG \& Co. KG \\ Karlsruhe, Germany \\ Email: johannes.kolb@schaeffler.com
}

\author{
Martin Doppelbauer \\ Elektrotechnisches Institut \\ Karlsruhe Institute of Technology \\ Karlsruhe, Germany \\ Email: martin.doppelbauer@kit.edu
}

\begin{abstract}
This contribution suggests an approach to determine the optimal design of a Permanent Magnet Synchronous Motor for a given application by calculating the effects of coil configuration switching, pole-changing and multi-phase windings. The impact on the torque-speed-characteristic of a motor is evaluated in a normalized parameter plane, enabling the designer to compare the influences by using criteria like the operating range. Moreover, a way of assessing additional semiconductors is introduced. The effects on an exemplary design are presented in a unified approach. Due to this, promising designs for the example can be identified, which double the reachable torquespeed area to nearly ideal values.
\end{abstract}

\section{INTRODUCTION}

With the emerge of electric and hybrid electric vehicles, the increase in power density and the decrease of installation space and weight to improve the cruising range is a driving topic in the design of electric motors [1], [2]. Permanent Magnet Synchronous Motors (PMSM) are a promising choice to achieve this [3]. Due to additional reluctance torque the field-weakening area can be adjusted in that way that a continuous torque speed ratio can be reached which fits the demands of traction drives best [4]. However, to match various constraints the designs are often limited to lower power densities or operating ranges. For example, the maximum allowed induced voltage decreases the maximum permitted permanent flux linkage or saturation reduces the available reluctance torque leading to higher space requirements. With this in mind, efforts were undergone to find ways to improve the PMSM further: Approaches known from grid-connected motors are also proposed for traction drives, as for example star-delta-configuration changes [5] or pole-changing [6] to expand the operating area of these motors. The possibilities of coil switching are extended in [7]. For pole-changing, there are two major approaches to implement the pole-change in the stator winding: on the one hand a winding configuration like a Dahlander winding enabling a simplified change of the pole number of the stator field [8] and on the other hand a change in the phase number leading automatically to a different pole-number. For changing the pole number in the rotor, [6] introduced the possibility to use demagnetizable magnets, which can be magnetized in that way, that the favored pole number can be achieved. It could be also done by electrically excited rotor poles, which would however lead to different motor types aside from PMSMs. Thus, it is not considered in this contribution.

Additionally, multi-phase motors with a phase number $m$ greater than three are discussed as well because of their improved performance and fault-tolerance [9]-[11]. The advantage of a multi-phase winding compared to a three-phase winding consists in an improved usage of the DC-link voltage at space vector pulse width modulation (SVPWM) [9]. With the same DC-link voltage, the reachable phase voltage can be improved by up to $10 \%$ while the peak-to-peak-value of the common mode voltage converges to zero for increasing $m$ [9] leading to reduced capacitive leakage currents [12]. Moreover, the performance of the motor itself is improved as the coupling between rotor and stator is enhanced [9].

These suggestions to improve a PMSM are considered mostly isolated in the contributions and it is therefore hard to compare the results. This is especially the case if an optimal design is demanded under certain given constraints as the number of stator slots or the maximum induced voltage or the maximum short-circuit currents. The remedy hereof is the adaption of the parameter plane for these configuration changes which is described in this paper. This enables the designer to compare each consequence of coil configuration switching, pole-changing or multi-phase winding in relation to each other as it is schematically given in Fig. 1. The parameter plane itself proposed by [4] is an ideal tool to determine analytically the torque-speed-characteristics of motors. It allows the prediction by only two parameters, the permanent magnet flux linkage $\psi_{\mathrm{PM}}$ and the saliency $\zeta$, which is the ratio of the quadrature inductance $L_{\mathrm{q}}$ to the direct inductance $L_{\mathrm{d}}$.

In this contribution, the parameter plane is first described shortly. In the following section, the adaption of the original parameter plane is submitted, which is necessary in order to actually see the changes in the right relation to each other. Afterwards, the effects of the constraints due to short-circuit current and induced voltage at maximum speed are given. The 

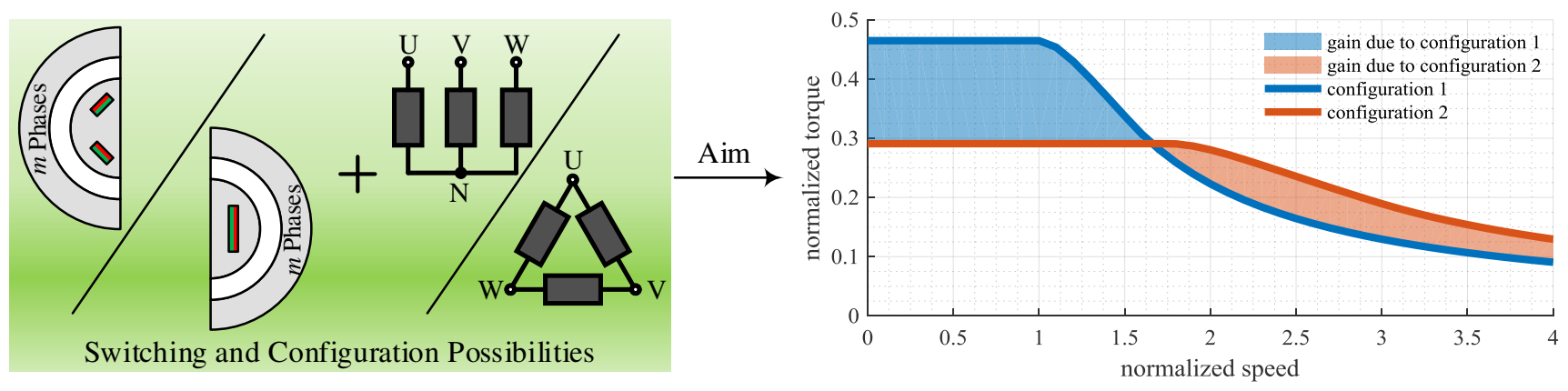

Figure 1. The considered different change and switching options of PMSM are displayed in a schematic way. The aim is to achieve a greater operating area due to pole-changing, a higher phase number and / or coil configuration switching which is exemplarily presented in the diagram on the right hand side.

next section consists of the description of an exemplary design in order to show the application of the proposed equations. The results are introduced thereafter and set in relation to an increased effort in semiconductors.

\section{The PARAmeter Plane}

In order to establish the parameter plane, the following assumptions have to be made [4]:

- the stator resistance is set to zero

- the losses in the iron and the magnet and due to friction are neglected

- saturation which would change the inductances is not taken into account

- only the fundamental wave is considered leading to a sinusoidal excitation

Furthermore, using the Maximum Torque per Ampere (MTPA) and Maximum Torque per Voltage (MTPV) procedures [2] with the conditions for the maximum current $i_{\max }$, the maximum voltage $v_{\max }$

$$
\begin{gathered}
\sqrt{v_{\mathrm{d}}^{2}+v_{\mathrm{q}}^{2}} \leq v_{\max }=1 \\
\sqrt{i_{\mathrm{d}}^{2}+i_{\mathrm{q}}^{2}} \leq i_{\max }=1
\end{gathered}
$$

the torque-speed-characteristic can be determined for each tuple of $\psi_{\mathrm{PM}}$ and $\zeta$. The values are normalized in that way that the maximum current $i_{\max }$, the maximum voltage $v_{\max }$, the maximum ideal torque $t$ and power are set to one in order to be able to compare different motor designs. That means that the maximum base speed is also normalized to one and the field weakening area starts at speed $\omega=1$. That leads to following equations [4]:

$$
\begin{gathered}
v_{\mathrm{d}}=-\omega \zeta l_{\mathrm{d}} i_{\mathrm{q}} \\
v_{\mathrm{q}}=\omega l_{\mathrm{d}} i_{\mathrm{d}}+\omega \psi_{\mathrm{PM}} \\
t=\psi_{\mathrm{PM}} i_{\mathrm{q}}-(\zeta-1) l_{\mathrm{d}} i_{\mathrm{d}} i_{\mathrm{q}}
\end{gathered}
$$

where $l_{\mathrm{d}}$ is the normalized direct inductance, $v_{\mathrm{d}}$ and $v_{\mathrm{q}}$ are the normalized voltages in $\mathrm{d}$ - and q-axis and $i_{\mathrm{d}}$ and $i_{\mathrm{q}}$ are the normalized currents in the specified axes.

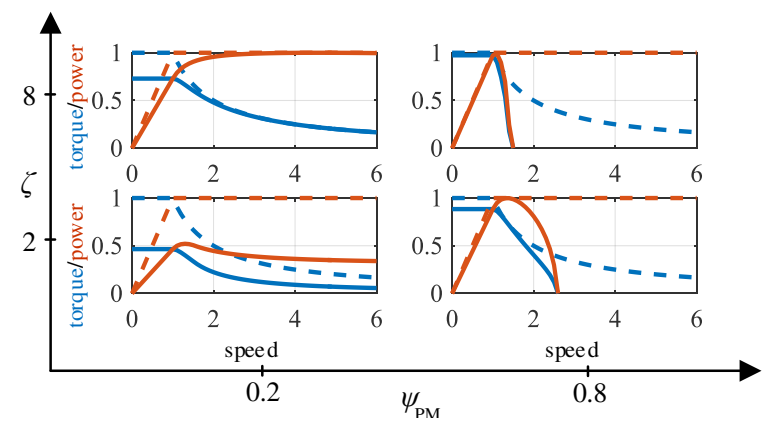

Figure 2. Normalized power - torque - characteristics in the parameter plane dependent on $\psi_{\mathrm{PM}}$ and $\zeta$ with the ideal characteristics in dashed lines [7]

[4] showed that the motor equations consist of only two independent parameters: $\psi_{\mathrm{PM}}$ and $\zeta$, since $l_{\mathrm{d}}$ is dependent of $\psi_{\mathrm{PM}}$ and $\zeta$ and can therefore be calculated. $\psi_{\mathrm{PM}}$ and $\zeta$ consequently span a parameter plane, where the motor characteristics are presented as shown in Fig. 2 [7].

\section{The Extension of the Parameter Plane}

There are a few examples, where the parameter plane has been adapted to specific issues as for example a variable flux [13], different supply voltage and current ratios [14] or winding configurations [7], [15]. In this contribution, the variables taken into account are the phase number $m$, the number of windings in series per phase $w$, the winding factor $\xi$ and the number of pole pairs $p$. The equations are then normalized to the base values of these variables enabling setting the right relation to each of the different torque-speedcharacteristics, as it was done for $w$ in [7].

Under the condition, that the inductances of the different phases are symmetrical and the coupling between the phases is identical, the following equation is valid [8]:

$$
L_{\mathrm{d}, \mathrm{q}}=\frac{m}{2} \mu_{0} C_{\mathrm{d}, \mathrm{q}} \frac{1}{\delta} \tau_{\mathrm{p}} l_{\mathrm{Fe}} \frac{w^{2} \xi^{2}}{p}
$$

where $\delta$ is the air gap, $\tau_{\mathrm{p}}$ the pole pitch, $l_{\mathrm{Fe}}$ the stack length and $C_{\mathrm{d}}$ and $C_{\mathrm{q}}$ describe the relation of the flux density amplitude to the magneto motive force in the direct or quadrature axis. Moreover, it is assumed, that $C_{\mathrm{d}, \mathrm{q}}$ do not change during 
pole-changing, because of a uniform air gap and the magnets' assembly. That means that the dependence of the absolute inductances $L_{\mathrm{d}, \mathrm{q}}$ can be written as $L_{\mathrm{d}, \mathrm{q}} \propto m \cdot w^{2} \cdot \xi^{2} \cdot \frac{1}{p^{2}}$. As $\psi_{\mathrm{PM}}$ can be calculated by [8]

$$
\psi_{\mathrm{PM}}=\frac{2}{\pi} w \xi \hat{B}_{\mathrm{PM}} \tau_{\mathrm{p}} l_{\mathrm{Fe}}
$$

$\psi_{\mathrm{PM}}$ is proportional to $w \cdot \xi \cdot \frac{1}{p}$. $\hat{B}_{\mathrm{PM}}$ is the amplitude of the flux density of the permanent magnet.

Since the aim is to depict the exact relation of the change in the phase number, coil configuration or pole number, factors are introduced describing the change in those variables compared to the base value set as following:

- the ratio factor $k_{\mathrm{w}}$ describes the change in the coil configuration with $k_{\mathrm{w}}=\frac{w}{w_{\mathrm{b}}}$ with the base winding number $w_{\mathrm{b}}$ and $w \leq w_{\mathrm{b}}$

- the ratio factor $k_{\xi}$ describes the change in the winding factor with $k_{\xi}=\frac{\xi}{\xi_{\mathrm{b}}}$ with the base winding factor $\xi_{\mathrm{b}}$ and $\xi \leq \xi_{\mathrm{b}}$

- the ratio factor $k_{\mathrm{p}}$ describes the change in the pole pair number with $k_{\mathrm{p}}=\frac{p_{\mathrm{b}}}{p}$ with the base pole pair number $p_{\mathrm{b}}$ and $p \geq p_{\mathrm{b}}$

- the ratio factor $k_{\mathrm{m}}$ describes the change in the phase number with $k_{\mathrm{m}}=\frac{m}{m_{\mathrm{b}}}$ with the base phase number $m_{\mathrm{b}}$ and $m \leq m_{\mathrm{b}}$

With the chosen normalization, the ratio factors can attain values between $0<\left.k_{\mathrm{x}}\right|_{x=w, \xi, p, m} \leq 1$. Consequently, the equations eq. (3) to eq. (5) can be written as:

$$
\begin{gathered}
v_{\mathrm{d}}=-k_{\mathrm{w}}^{2} k_{\xi}^{2} k_{\mathrm{p}}^{2} k_{\mathrm{m}} \omega \zeta l_{\mathrm{d}} i_{\mathrm{q}} \\
v_{\mathrm{q}}=k_{\mathrm{w}}^{2} k_{\xi}^{2} k_{\mathrm{p}}^{2} k_{\mathrm{m}} \omega l_{\mathrm{d}} i_{\mathrm{d}}+k_{\mathrm{w}} k_{\xi} k_{\mathrm{p}} \omega \psi_{\mathrm{PM}} \\
t=\frac{k_{\mathrm{w}} k_{\xi} k_{\mathrm{p}} \psi_{\mathrm{PM}} i_{\mathrm{q}}-k_{\mathrm{w}}^{2} k_{\xi}^{2} k_{\mathrm{p}}^{2} k_{\mathrm{m}}(\zeta-1) l_{\mathrm{d}} i_{\mathrm{d}} i_{\mathrm{q}}}{k_{\mathrm{p}} k_{\mathrm{m}}}
\end{gathered}
$$

There is no further need for adaption concerning $w$ and $\xi$ but it is necessary to undertake an additional step for changing the phase number or the pole number which can be seen in eq. (10). In the parameter plane, the normalized frequency that is used is the electric one. This leads to an inevitable adjustment of $\omega$ by $k_{\mathrm{p}}$ if the mechanical speed shall be depicted for a better comparison of the characteristics. The torque has to be adapted in the same course by $1 / k_{\mathrm{p}}$ (cf.eq. (10)). If the number of phases is changed from three to $m$, either the number of windings per coil has to be increased by $m / 3$ to get the same winding number $w$ at a reduced phase current $i_{\max }=i_{\max , \mathrm{b}} / k_{\mathrm{m}}$ or $w$ is automatically decreased if the winding of the coils stays the same leading to a reduction of the terminal voltage $v_{\max }=v_{\max , \mathrm{b}} / k_{\mathrm{m}}$ for a comparable power output. Due to a higher phase number, the torque has to be also multiplied by $m$ or divided by $k_{\mathrm{m}}$ respectively (cf.eq. (10)). Even though the number of variables increases, the introduction of the ratio factors enables a fast way to assess the change in the torque-speed-characteristic.

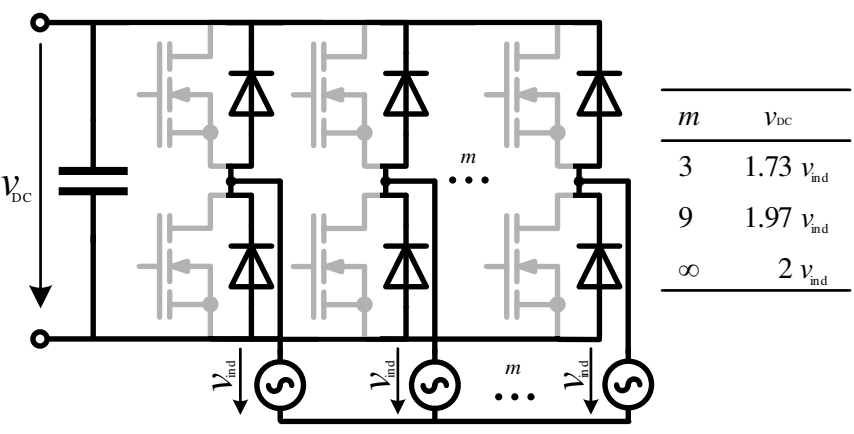

Figure 3. The resulting diode rectifier if an $m$ phase bridge is used and no gate signals are applied. The DC-link voltage icreases with the phase number $m$ at the same induced phase voltages.

\section{Constraints in the Parameter Plane}

Two safety measures dominating the design of PMSM are the limitation of the short circuit current and the induced voltage at maximum speed. The short circuit current has to be limited because the produced heat by power dissipation must not destroy the motor or the resulting torque has to be restricted to not damage mechanical parts of the power train. If the induced voltage increases the DC link voltage above the maximum blocking voltage of the semiconductors $v_{\mathrm{br}}$, this would lead to a destruction of them. Both criteria restrict the design space in the parameter plane. As the stator resistance is set to zero for the calculation, both limits can be calculated easily by eq. (3) and eq. (4). The short circuit current $i_{\mathrm{sc}}$ results in

$$
i_{\mathrm{sc}}=\frac{\psi_{\mathrm{PM}}}{l_{\mathrm{d}}}
$$

by setting the voltage to zero. The equation for the maximum induced voltage $v_{\text {ind }}$ can be written as

$$
v_{\text {ind }}=\omega_{\max } \psi_{\mathrm{PM}} \sqrt{(2(1-\cos (\gamma)))}
$$

whereby the angle $\gamma$ can be calculated by $\gamma=\frac{m-1}{m} \pi$ for normal multi-phase systems assuming that a m-phaseleg voltage source inverter is used and that it is working as a diode rectifier when no gate signals are applied to the semiconductors. Fig. 3 depicts this scenario with MOSFETs as switches. Fig. 4 shows the design space in the parameter plane reduced by both constraints at exemplary values. The red colored space cannot be used anymore if $i_{\mathrm{sc}} \leq i_{\max }$ (Fig. 4 a)) or if $v_{\text {ind, } \max }\left(\omega_{\max }=3\right) \leq 1.3 v_{\max }$ (Fig. 4 b)) respectively. As can be seen, the possible design space is diminished significantly reducing the reachable maximum base torque strongly.

If the base motor design is changed or a motor parameter can be switched, the constraints are altered as well. Applying the switching ratios $\left.k_{\mathrm{x}}\right|_{x=w, \xi, p, m}$ to eq. (11) and eq. (12), this results in

$$
i_{\mathrm{sc}, \mathrm{k}}=\frac{1}{k_{\mathrm{m}} k_{\mathrm{w}} k_{\xi} k_{\mathrm{p}}} \frac{\psi_{\mathrm{PM}}}{l_{\mathrm{d}}}
$$



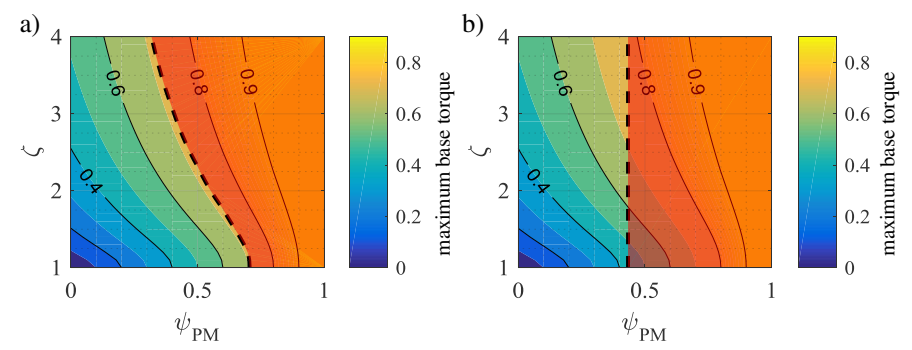

Figure 4. Constraints in the parameter plane (dashed line) displaying the maximum torque in the base speed area and the restriction due to a) $i_{\mathrm{SC}} \leq$ $i_{\max }$ or $\left.\mathrm{b}\right) v_{\text {ind }} \leq 1.3 u_{\max }$ with $\omega_{\max }=3$. The non-usable space is colored red.

$$
v_{\text {ind }, \mathrm{k}}=k_{\mathrm{w}} k_{\xi} \omega_{\max } \psi_{\mathrm{PM}} \sqrt{2\left(1-\cos \left(\frac{m-1}{m} \pi\right)\right)}
$$

implying that an increased effective winding number $w$, winding factor $\xi$, phase number $m$ and a smaller pole pair number $p$ lead to lower short-circuit currents $i_{\mathrm{sc}}$. However, the maximum induced voltage $v_{\text {ind }}$ decreases if $w, \xi$ and $m$ are diminished.

\section{Application of the EXtension - EXAmple Designs}

To illustrate the usability of the extended parameter plane and the advantages and disadvantages of the concept of a switchable motor, an exemplary PMSM is designed. This shows cause and effect of changing the mentioned motor parameters, $m, w, \xi$ and $p$, where $\psi_{\mathrm{PM}}$ and the inductances $l_{\mathrm{d}}$ and $l_{\mathrm{q}}$ are normalized to the maximum base values. The slot number $N$ is set to 36 , which holds enough possibilities for the switching with a common slot number. It includes two normal multi-phase systems $m=3$ and $m=9$, leading to the following options, which are assessed in section VI:

- option 1: a distributed winding with $p=2$ and $m=3$, leading to $\xi=0.96$

- option 2: a distributed winding with $p=2$ and $m=9$, leading to $\xi=1$

- option 3: a switching coil configuration with $p=2$ and $m=3$ holding 4 different coil configurations $k_{\mathrm{w}}=1,1 / \sqrt{3}, 1 / 2,1 /(2 \sqrt{3})$ [7]

- option 4: a switching coil configuration with $p=2$ and $m=9$ holding 10 different coil configurations $k_{\mathrm{w}}=$ $1,0.684,0.532,0.5,0.395,0.347,0.342,0.266,0.197$, 0.174 [7]

- option 5: a pole-change with a $\Delta$-YY-Dahlander-winding from $p=3$ to $p=6$ at $m=3$, leading to $\xi(p=3)=$ 0.683 and $k_{\mathrm{w}}=\sqrt{3} / 2$ and $\xi(p=6)=1$ and $k_{\mathrm{w}}=1$

For the examination, $\psi_{\mathrm{PM}}$ can at first be chosen between 0 and 1 , while the range of $\zeta$ is constrained at the interval 1 to 3 . $\zeta_{\max }=3$ is chosen since it is the maximum saliency achievable for PMSMs with a single barrier interior PM considering saturated saliency ratios [4]. This makes sure that the evaluated designs are located in a usable design space holding the maximum reachable torque-speed-characteristics. For the comparison of the designs, those characteristics are
Table I

CONSTANT PARAMETERS FOR ALL EXAMPLE DESIGNS

\begin{tabular}{ll}
\hline Design Parameter & Value \\
\hline$N$ & 36 \\
$v_{\text {ind, } \max }$ & $1.3 v_{\max }$ \\
$i_{\mathrm{sc}, \max }$ & $i_{\max }$ \\
$n_{\max }$ & 6 \\
$m$ & 3 or 9 \\
\hline
\end{tabular}

matched against each other defining the performance of each configuration.

Regarding pole-changing, eq. (10) means that the reluctance torque is reduced for higher pole pair numbers while the synchronous torque stays constant. The base maximum torque and the field weakening ability for $k_{\mathrm{p}}<1$ is consequently decreased against $k_{\mathrm{p}}=1$. This is valid if no saturation occurs or both designs are saturated evenly. Otherwise, the desaturation of the flux paths of $k_{\mathrm{p}}<1$ leading to higher inductances counters its negative effects on the reluctance torque and the field weakening ability. Since this is strongly dependent on the chosen geometric design and the used materials, this case is not considered in this paper for keeping a more general approach. Therefore, the pole-phase-modulation is not calculated as well because the proposed winding setup in [16] contains the same winding number and winding factor for both configurations. This means that a difference between the configurations can only be seen in the pole pair number leading to a reduced performance of higher $p$ but no spreading of the torque-speed-area.

Moreover, the maximum values of current $i_{\max }$, voltage $v_{\max }$, induced voltage $v_{\text {ind, } \max }=1.3 v_{\max }$ and short-circuit current $i_{\mathrm{sc} \text {, max }}=i_{\max }$ are given. For switchable configuration designs, $\psi_{\mathrm{PM}, \max }$ can be chosen higher than for non switchable designs. Keeping for example eq. (14) in mind, a reduction in $w$ due to another coil configuration leads to smaller induced voltages at the same maximum speed. The switching is done by bidirectional semiconductor switches (cf. Fig. 11), which are carried out in such way that they automatically go in the state of the smallest induced voltage in case of a lack of gate signals. For the examined designs, it is assumed that the $i_{\mathrm{sc} \text {, max }}$ must be satisfied if the motor is deliberately brought to the short circuit state by shorting all phases. This means that the inverter and the semiconductors are still controllable. Therefore, it is also possible to get in the state with the lowest short-circuit current. Hence, the constraints shown in Fig. 4 can be avoided or at least extended for switchable configurations which increases the available design space.

The maximum speed shall be $n_{\max }=6$. The set parameters which stay constant for each design configuration are summarized in Tab. I.

\section{Results of the Parameter Plane}

In the previous sections, the possible designs and the design constraints were introduced. In this section, the results of the parameter plane are discussed. To assess the results, the 


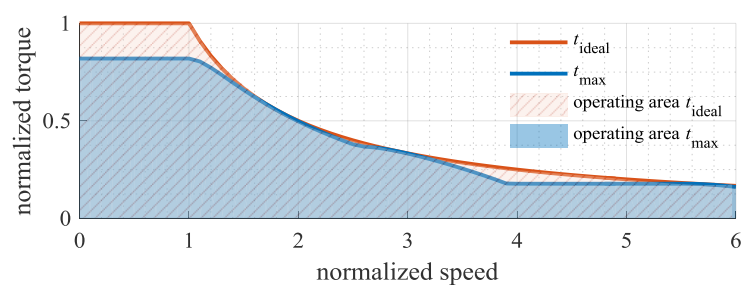

Figure 5. The area of the torque $\left(t_{\max }\right)$-speed-curve of an exemplary configuration and the area of the ideal characteristic illustrating the calculation of $A_{\mathrm{Pn}}$

operating-range-criterion $A_{\mathrm{Pn}}$ is used. It describes the area of the resulting torque-speed-characteristic which is compared to the area of the ideal torque-speed-characteristic [7], leading to the equation:

$$
A_{\mathrm{Pn}}=\frac{\int_{0}^{\omega_{\text {end }}} t_{\text {max }}\left(\omega,\left.k_{\mathrm{x}}\right|_{x=w, \xi, p, m}\right) \mathrm{d} \omega}{\int_{0}^{\omega_{\text {end }}} t_{\text {ideal }}(\omega) \mathrm{d} \omega}
$$

whereas $t_{\max }$ is the maximum torque concerning $k_{\mathrm{x}}$ for one design at a specific speed $\omega$ and $t_{\text {ideal }}$ is the torque of an ideal motor. $A_{\mathrm{Pn}}$ is therefore always smaller or at least the same as one. The calculation is depicted graphically in Fig. 5. In the following, the results for the given exemplary motor is discussed.

\section{A. Comparison of Option $1(m=3)$ to Option $2(m=9)$}

The relative difference in $A_{\mathrm{Pn}}, \Delta A_{\mathrm{Pn}, \mathrm{rel}}$, of $m=3$ and $m=9$ is shown in Fig. 6. This is valid, if the shortcircuit and maximum induced voltage constraints are not taken into account. It can be seen, that for every base motor design, the 9-phase motor dominates the 3-phase one. The operating range can be increased by $2 \%$ to $6 \%$ with the same amount of permanent magnets and copper. The 9 -phase motor additionally benefits by the improved usage of the DClink voltage. Considering the induced voltage and short-circuit current, the result changes. Tab. II lists $\psi_{\mathrm{PM} \text {,max }}$ which is calculated due to the maximum allowed DC-link voltage for $m=3$ and $m=9$. Clearly, there is a disadvantage for $m=9$ since the interlinking factor of the diode rectifier for $m=9$ is nearly ideal compared to $m=3$, which increases the DC-link voltage at the same phase voltage by nearly $14 \%$ compared to $m=3$ (cf. Fig. 3). Therefore, only small $n_{\max }$ do not constrain the parameter plane due to the maximum induced voltage and the results of Fig. 6 stay. The result for $n_{\max }=6$ is depicted in Fig. 7a) with the dashed curves. These curves are calculated at $\left.\psi_{\mathrm{PM}, \max }\right|_{\mathrm{m}=3}$ and $\left.\psi_{\mathrm{PM}, \max }\right|_{\mathrm{m}=9}$. The 9 -phase design reaches nearly the 3 -phase design, although $m=3$ needs more $\psi_{\mathrm{PM}}$ do reach that. If the same rotor design with $\psi_{\mathrm{PM}} \leq\left.\psi_{\mathrm{PM}, \max }\right|_{\mathrm{m}=9}$ is used, $m=9$ dominates $m=3$ regarding $A_{\mathrm{Pn}}$ completely. The solid curves in Fig. 7a) represent the short-circuit constraint. Here, the 9-phase motor design is advantageous since a smaller $\xi$ increases the short circuit current and the tuple of $\psi_{\mathrm{PM}}$ and $\zeta$ therefore has to be decreased for $m=3$. All in all, it can be concluded that with

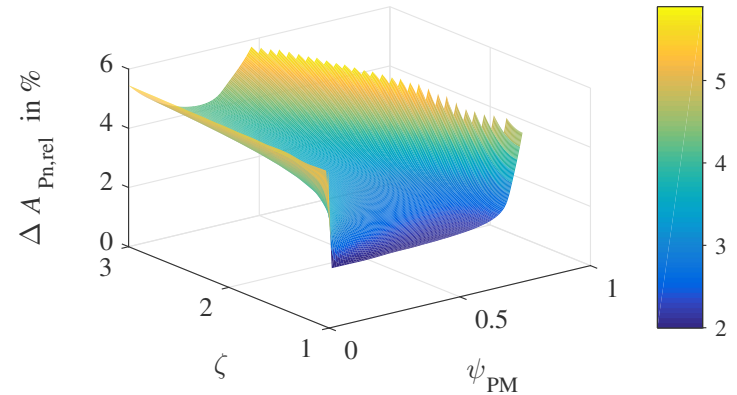

Figure 6. $\psi_{\mathrm{PM}}-\zeta-\Delta A_{\mathrm{Pn}, \mathrm{rel}}$-map describing the relative augment of $A_{\mathrm{Pn}}$ of $m=9$ compared to $m=3$ in $\%$ without additional constraints like short-circuit-current or induced voltage

Table II

$\psi_{\mathrm{PM}, \max }$ FOR $m=3(\xi=0.96)$ AND $m=9(\xi=1)$

\begin{tabular}{lll}
\hline$n_{\max }$ & $\left.\psi_{\mathrm{PM}, \max }\right|_{\mathrm{m}=3}$ & $\left.\psi_{\mathrm{PM}, \max }\right|_{\mathrm{m}=9}$ \\
\hline 1 & 1 & 1 \\
2 & 0.6771 & 0.5716 \\
4 & 0.3250 & 0.2858 \\
6 & 0.2257 & 0.1905 \\
9 & 0.1505 & 0.1270 \\
\hline
\end{tabular}

the same $\psi_{\mathrm{PM}}$ the $m=9$ motor is always superior in $A_{\mathrm{Pn}}$ to the $m=3$ motor.

\section{B. Comparison of Option 3 ( $m=3$, 4 coil config.) and Option 4 ( $m=9$, 10 coil config.) to Option $1(m=3)$}

In accordance to [7], there are four coil configurations for the 3 -phase motor with $p=2$ and a distributed winding. The coils could be arranged in series or in parallel configuration and in a star- or delta-connection. Other parallel configurations are conceivable, but this would lead to circulating currents since the induced voltage in the parallel coils is phase shifted. If this design is done with nine phases, the number of configurations rises up to ten, as there are four polygon-configurations additional to the star-configuration and a series-parallel connection [7]. For this comparison, only the maximum amount of switchings is considered in order to get the maximum effects. In fact, lesser switching numbers could result in similar $A_{\mathrm{Pn}}$ at a distinct tuple of $\psi_{\mathrm{PM}}$ and $\zeta$.

The outcome of this comparison regarding the difference of coil configuration switching to fixed $m=3$ configuration is shown in Fig. 7b). Hereby, all configurations abide by $v_{\text {ind,max }}$. It is clearly visible, that the reduced induced voltage due to switchings, which was described in the previous section, leads to a big improvement of $A_{\mathrm{Pn}}$. Besides, the 9-phase design dominates the 3 -phase in contrast to no coil switching in Fig. 7a) and shows a nearly ideal operating range. If the short-circuit current is additionally included, the depiction of Fig. 7c) arises.

\section{Comparison of Option 5 (pole-changing $m=3$ ) to Option $1(m=3)$}

In order to describe the effects of the pole-changing with a Dahlander-winding in particular, the result of $\psi_{\mathrm{PM}}=0.65$ 

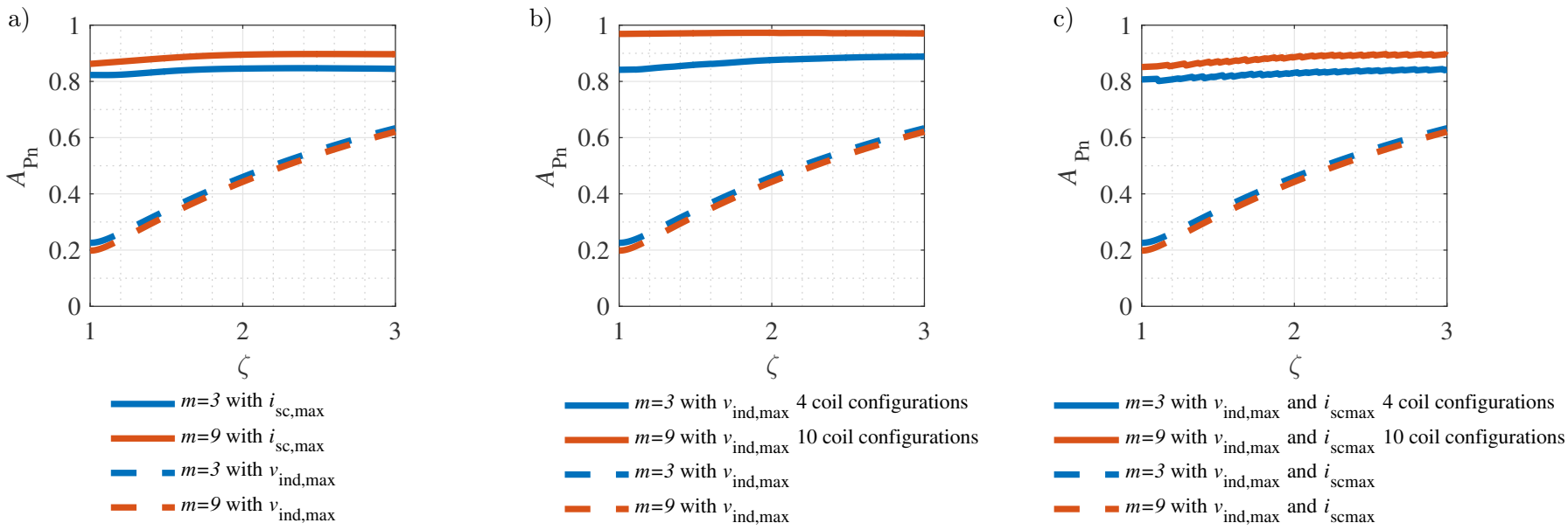

Figure 7. $\zeta-A_{\mathrm{Pn}}$-diagram, describing $A_{\mathrm{Pn}, \max }$ of $m=9$ compared to $m=3$ at a distinct $\zeta$, whereby $v_{\text {ind,max }}$ or $i_{\mathrm{sc}, \max }$ are included in a), coil switching is compared to a fixed configuration under the condition $v_{\text {ind,max }}$ in b) and coil switching is compared to a fixed configuration under the conditions $v_{\text {ind,max }}$ and $i_{\mathrm{sc}, \max }$ in $\mathrm{c}$ ).

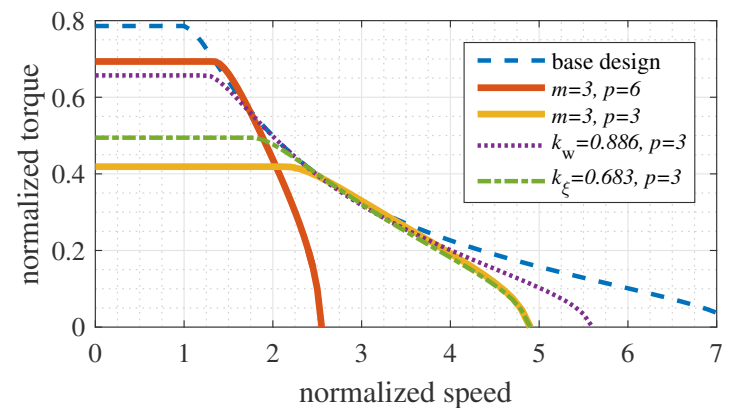

Figure 8. Torque-speed-diagram of $\psi_{\mathrm{PM}}=0.65$ and $\zeta=2$ displaying what happens with pole-changing motors using a Dahlander-winding

and $\zeta=2$ is displayed in Fig. 8 for $p=3$ and $p=6$. An obvious change of the torque-speed-characteristic is visible and the operating range of $p=6$ is spread by the $p=3$ design. However, if $p=6$ is compared to the base design (dashed line) with $p=3$ with $k_{\xi}=1$ and $k_{\mathrm{w}}=1$ instead of $k_{\xi}=0.683$ and $k_{\mathrm{w}}=0.866$, there is no gain in $A_{\mathrm{Pn}}$. On the contrary, the maximum base speed is hardly changed but the maximum achievable speed and the field-weakening ability is reduced. The spreading is achieved because of the different attributes of the Dahlander-winding. As mentioned in the previous section, there is an antagonist, if saturation is applied and therefore flux paths are desaturated at higher pole pair numbers. Aside from that, the big drawback of the pole-changing, even though no disadvantages due to the rotor commutation is assumed, is the deterioration in the winding factor and in the reachable winding number with the used Dahlander winding, leading to the results in Fig. 9. In this comparison, the 3 -phase design with $p=2$ is clearly superior if no constraints are considered. Fig. 10 shows the influence of $v_{\text {ind,max }}$ and $i_{\mathrm{sc}, \max }$. Still the Dahlander-pole-changing cannot reach the results of the 3-phase motor with a fixed pole-pair number.

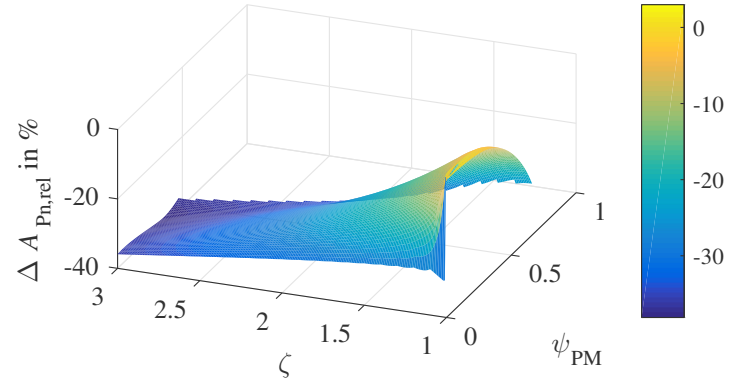

Figure 9. $\psi_{\mathrm{PM}}-\zeta-\Delta A_{\mathrm{Pn}, \mathrm{rel}}$-map describing the relative decline of $A_{\mathrm{Pn}}$ of the pole-changing Dahlander design compared to $m=3$ without additional constraints like short-circuit-current or induced voltage

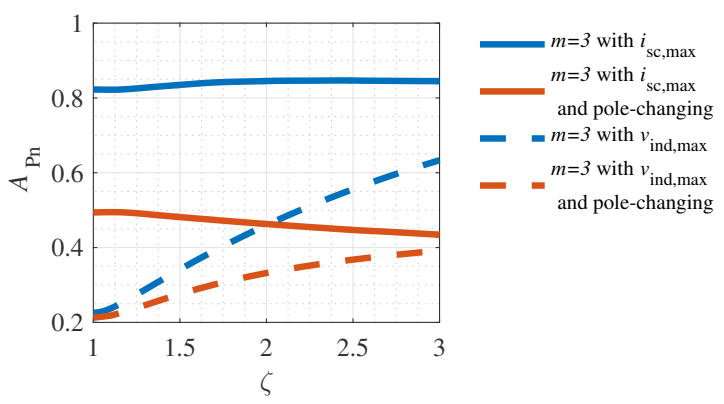

Figure 10. $\zeta$ - $A_{\mathrm{Pn}}$-diagrams, describing $A_{\mathrm{Pn}, \max }$ of the pole-changing Dahlander design compared to $m=3$ at a distinct $\zeta$ with $v_{\text {ind,max }}$ and $i_{\mathrm{Sc}, \max }$

\section{EXPENSES IN SEMICONDUCTORS}

As the different coil configurations of the motor have to be switched during operation to achieve the shown graphs, a switching unit is needed. Independent of the construction of the switch, each switch has to meet certain requirements $v_{\mathrm{br}}, i_{\max }$, normally on or off. As one possible implementation, MOSFETs have been chosen for this paper. Keeping in mind, that these have to be reverse blocking switches, two MOSFETs 


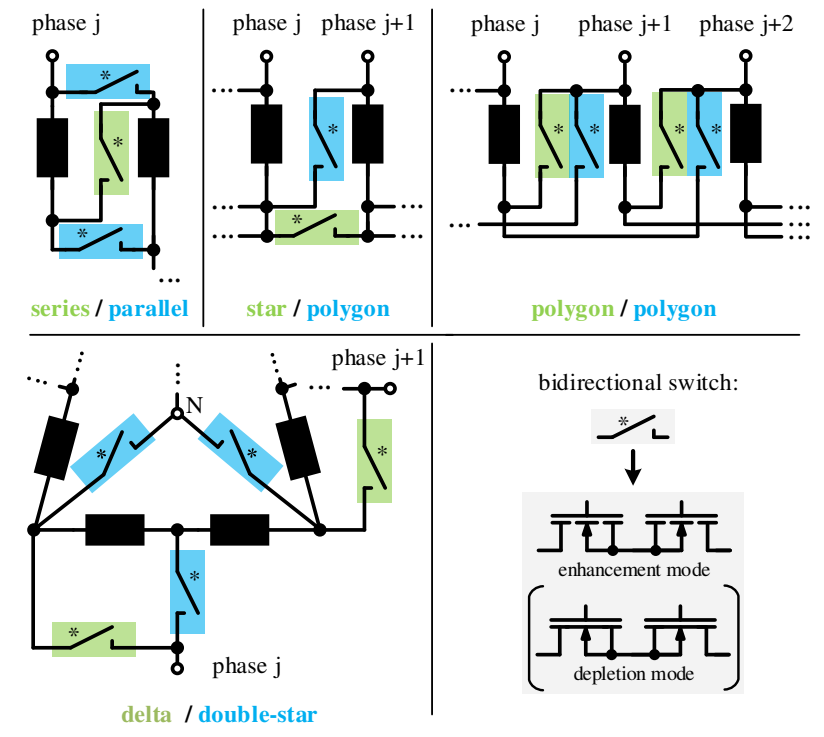

Figure 11. Assumed switching topologies for the different coil switching configurations. A half bridge as in Fig. 3 is connected to each phase connection point $\mathrm{j}$.

Table III

NEEDED SWITCHES FOR DIFFERENT CONFIGURATIONS

\begin{tabular}{ll}
\hline Switching Configuration & $n_{\mathrm{bs}}$ \\
\hline Series/Parallel (Ser / Par) & $3 m$ \\
Star/Polygon (Y / PG) & $2 m-1$ \\
Polygon/Polygon (PG / PG) & $2 m$ \\
Delta/Double-Star ( $\Delta$ /YY) & $3 m$ \\
\hline Ser / Par / Y / PG & $3 m+(2 m-1)$ \\
Ser / Par / Y / 4PG & $8 m-1$ \\
Y / 4PG & $5 m-1$ \\
\hline
\end{tabular}

have to be connected anti-serially to form one bidirectional switch. This allows the calculation of the expenses of the switching by using growth laws based on [17]. For this contribution, a volume criterion $V_{\text {MOSFET }}$ of semiconductors is used [17]:

$$
V_{\mathrm{MOSFET}}=2.5\left(\frac{v_{\mathrm{br}}}{v_{\mathrm{br}, \mathrm{b}}}\right)^{1.8}\left(\frac{I_{\mathrm{rms}, \max }}{I_{\mathrm{rms}, \max , \mathrm{b}}}\right)^{1}
$$

$v_{\mathrm{br}, \mathrm{b}}$ and $I_{\mathrm{rms}, \max , \mathrm{b}}$ are the maximum blocking voltage and the maximum semiconductor current used as a reference semiconductor. $v_{\mathrm{br}}$ is the actual blocking voltage and $I_{\mathrm{rms}, \max }$ is the actual maximum current of the semiconductor which should be compared. The volume change of diodes can be calculated due to dividing $V_{\text {MOSFET }}$ by 5 [17]. The basis is the 3-phase bridge (3PB) and its needed semiconductor values with the volume expense $V_{3 \mathrm{~PB}}$

$$
V_{3 \mathrm{~PB}}=\underbrace{6 V_{\text {MOSFET }}}_{6 \text { MOSFETs }}+\underbrace{6 \cdot \frac{V_{\text {MOSFET }}}{5}}_{6 \text { Diodes }}=7.2 V_{\text {MOSFET }}
$$

as depicted in Fig. 3. Additional diodes are used since the body diodes of MOSFETs usually produce high losses. If the phase number is tripled (cf. Fig. 3) and therefore the DC-link
Table IV

$m=3$ PMSM WITH 4 COIL CONFIGURATIONS OR POLE-CHANGING

\begin{tabular}{llll}
\hline Switching & State & $v_{\mathrm{br}}$ & $I_{\mathrm{rms}, \max }$ \\
\hline Ser / Par / Y / PG & Ser & $v_{\mathrm{br}, \mathrm{b}}$ & $I_{\mathrm{rms}, \max , \mathrm{b}} / 2$ \\
Ser / Par / Y / PG & $\mathrm{Par}$ & $v_{\mathrm{br}, \mathrm{b}} / 2$ & $I_{\mathrm{rms}, \max , \mathrm{b}} / 2$ \\
Ser / Par / Y / PG & $\mathrm{Y}$ & $v_{\mathrm{br}, \mathrm{b}}$ & $I_{\mathrm{rms}, \max , \mathrm{b}}$ \\
Ser / Par / Y / PG & $\mathrm{PG}$ & $v_{\mathrm{br}, \mathrm{b}} / \sqrt{3}$ & $I_{\mathrm{rms}, \max , \mathrm{b}} / \sqrt{3}$ \\
\hline$\Delta / \mathrm{YY}$ & $\Delta$ & $v_{\mathrm{br}, \mathrm{b}} / \sqrt{3}$ & $I_{\mathrm{rms}, \max , \mathrm{b}}$ \\
$\Delta / \mathrm{YY}$ & $\mathrm{YY}$ & $v_{\mathrm{br}, \mathrm{b}} / 2, v_{\mathrm{br}, \mathrm{b}} / \sqrt{3}$ & $I_{\mathrm{rms}, \max , \mathrm{b}}$ \\
\hline
\end{tabular}

voltage is reduced, the 9-phase bridge (9PB) results in the volume expense $V_{9 \mathrm{~PB}}$

$$
\begin{aligned}
V_{9 \mathrm{~PB}} & =\underbrace{18 \cdot 1.2 V_{\text {MOSFFET }}}_{18}\left(\frac{1}{3}\right)^{1.8}(1)^{1} \\
& \approx \quad 2.99 V_{\text {MOSFET }} 18 \text { Diodes }
\end{aligned}
$$

If the DC-link capacitor is taken into account (it is assumed that electrolyte capacitors are used), the decrease in the DClink capacitor expense $V_{9 \mathrm{~PB}, \mathrm{C}}$ for a 9-phase bridge compared to a 3-phase bridge $V_{3 \mathrm{~PB}, \mathrm{C}}$ can be calculated as [17]:

$$
\begin{aligned}
V_{9 \mathrm{~PB}, \mathrm{C}} & =\left(\frac{v_{\mathrm{DC}, \mathrm{PB} 9}}{v_{\mathrm{DC}, \mathrm{PB} 3}}\right)^{1.5}\left(\frac{I_{\mathrm{rms}, \mathrm{c}}}{I_{\mathrm{rms}, \mathrm{c}, \mathrm{b}}}\right)^{1}=\left(\frac{1}{3}\right)^{1.5} \\
& \approx 0.192 V_{3 \mathrm{~PB}, \mathrm{C}}
\end{aligned}
$$

The switched coil configurations have additional semiconductors to the semiconductors in the $m$-phase bridges. Fig. 11 depicts the needed switches for those configurations. Compared to the MOSFETs in the $m$-phase bridge, no diodes are necessary because of the overlapped switching. The number of bidirectional switches $n_{\mathrm{bs}}$ is given in Tab. III. The bidirectional switches itself need divergent $v_{\mathrm{br}}$ and $I_{\mathrm{rms}, \max }$ dependent on all switching configurations and the switching state itself. They can be calculated by including the maximum phase voltage due to the modulation scheme (here: SVPWM) and the inherent motor voltages due to the different winding connections described by $k_{\mathrm{w}}$. The current has to be adapted by $k_{\mathrm{w}}$, too. Hence, reducing the number of switching configurations would also cause a decrease in needed $v_{\mathrm{br}}$ and/or $I_{\mathrm{rms} \text { max }}$. The results for the considered $m=3$ configurations are listed in Tab. IV and for the $m=9$ configurations in Tab. V. Using the following equation

$$
\begin{aligned}
& V_{m-\mathrm{PB}, \mathrm{Sw}}=\underbrace{x V_{\mathrm{MOSFET}}}_{m-\mathrm{PB}} \\
& +\underbrace{\sum 2 n_{\mathrm{bs}} \cdot 1.2\left(\frac{v_{\mathrm{br}}}{v_{\mathrm{br}, \mathrm{b}}}\right)^{1.8} \frac{I_{\mathrm{rms}, \text { max }}}{I_{\mathrm{rms}, \mathrm{max}, \mathrm{b}}} V_{\text {MOSFET }}}_{\text {number of coil configurations }}
\end{aligned}
$$

leads to the volume expense $V_{3 \mathrm{~PB}, \mathrm{Sw}}$ for the exemplary $m=3$ PMSM with 4 different coil configurations:

$$
\begin{aligned}
V_{3 \mathrm{~PB}, \mathrm{Sw}} & =\underbrace{7.2 V_{\mathrm{MOSFET}}}_{3 \mathrm{~PB}}+\underbrace{13.95 V_{\mathrm{MOSFET}}}_{4 \text { coil configurations }} \\
& \approx 21.15 V_{\text {MOSFET }}
\end{aligned}
$$


Table V

$m=9$ PMSM WITH 10 DIFFERENT COIL CONFIGURATIONS

\begin{tabular}{llll}
\hline Switching & State & $v_{\mathrm{br}}$ & $I_{\mathrm{rms}, \max }$ \\
\hline Ser / Par / Y / 4PG & Ser & $0.414 v_{\mathrm{br}, \mathrm{b}}$ & $1.46 I_{\mathrm{rms}, \max , \mathrm{b}}$ \\
Ser / Par / Y / 4PG & Par & $0.207 v_{\mathrm{br}, \mathrm{b}}$ & $0.73 I_{\mathrm{rms}, \max , \mathrm{b}}$ \\
Ser / Par / Y / 4PG & Y & $0.414 v_{\mathrm{br}, \mathrm{b}}$ & $I_{\mathrm{rms}, \max , \mathrm{b}}$ \\
Ser / Par / Y / 4PG & PG1 & $0.414 v_{\mathrm{br}, \mathrm{b}}$ & $1.46 I_{\mathrm{rms}, \max , \mathrm{b}}$ \\
Ser / Par / Y / 4PG & PG2 & $0.414 v_{\mathrm{br}, \mathrm{b}}$ & $0.78 I_{\mathrm{rms}, \max , \mathrm{b}}$ \\
Ser / Par / Y / 4PG & PG3 & $0.414 v_{\mathrm{br}, \mathrm{b}}$ & $I_{\mathrm{rms}, \max , \mathrm{b}} / \sqrt{3}$ \\
Ser / Par / Y / 4PG & PG4 & $0.414 v_{\mathrm{br}, \mathrm{b}}$ & $0.51 I_{\mathrm{rms}, \max , \mathrm{b}}$ \\
\hline
\end{tabular}

whereas $m=9$ PMSM with 10 different coil configurations produces the volume expense $V_{9 \mathrm{~PB}, \mathrm{Sw}}$

$$
V_{9 \mathrm{~PB}, \mathrm{Sw}}=\underbrace{2.99 V_{\mathrm{MOSFET}}}_{9 \mathrm{~PB}}+\underbrace{22.42 V_{\mathrm{MOSFET}}}_{10 \text { coil conifgurations }}
$$$$
\approx 25.41 V_{\mathrm{MOSFET}}
$$

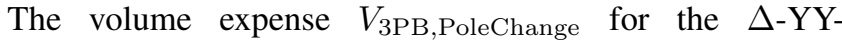
Dahlander-winding is calculated similarly and the needed data can be found in Fig. 11, Tab. III and Tab. IV as well. There is only one exception: The blocking voltage of the switches in YY-configuration has two different values. The three switches connecting the neutral point $N$ (cf. Fig. 11) need $v_{\mathrm{br}, \mathrm{b}} / \sqrt{3}$ whereas the other three need only $v_{\mathrm{br}, \mathrm{b}} / 2$

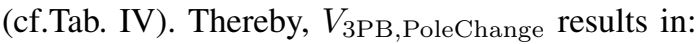

$$
\begin{aligned}
V_{3 \mathrm{~PB}, \text { PoleChange }} & =\underbrace{7.2 V_{\text {MOSFET }}}_{3 \mathrm{~PB}}+\underbrace{6.55 V_{\text {MOSFET }}}_{\Delta / \mathrm{YY}} \\
& \approx 13.75 V_{\text {MOSFET }}
\end{aligned}
$$

As can be seen, the needed semiconductor volume of a 9phase bridge is more than halved compared to a 3-phase bridge (cf. eq. (17), eq. (18)). Even if semiconductors with a higher blocking voltage are used to counter the better rectifier behavior of a 9-phase bridge and to increase the usable $\psi_{\mathrm{PM}}$, the volume of the semiconductors is still only half of that one of the 3-phase bridge. Moreover, the passive component for the 9-phase bridge is only a fifth of the 3-phase one (cf. eq. (19)).

The pole-changing motor does in contrast need a doubled expense in semiconductors (cf. eq. (23)) compared to a normal $m=3$ PMSM (cf. eq. (17)) without improving the behavior (cf. Fig. 10).

Coil configurations switching however yields actual gain in the operating range for every $m$ as can be seen in Fig. 7 b) and c) leading to motors with a higher power density and therefore reduced space requirements at the cost of a higher semiconductor volume. By choosing the number of configuration changes reasonably, one should be able to reduce the number of switches and thus the needed volume without deteriorating the gain in the operating range.

\section{CONCLUSION}

This contribution presents an analytical way to calculate the torque-speed-characteristics for pole-changing, coil switching and multi-phase PMSMs at given constraints, like the maximum allowed induced voltage and short-circuit currents. In this procedure, the higher expenditure of semiconductors at switchable configurations is evaluated additionally. As a result, it can be stated that pole-changing has a drastically reduced performance in contrast to a fixed 3-phase design if saturation effects do not occur. It is also shown that a spreading of the operating range is mostly not the consequence of the pole-change but of the change of the winding. Moreover, the supremacy of a 9-phase motor compared to 3-phase design is demonstrated and the gain in operating range by maximum $6 \%$ is calculated. Future work could focus on implementing saturation effects for pole-changing, on balancing the expansion of operating range by coil switching with the increasing of semiconductor volume or on taking power losses of the switches into account.

\section{REFERENCES}

[1] S. Rogers, U.S. Departement of Energy: (2017, April 25), Advanced Power Electronics and Electric Motors $R \& D$ [Online]. Available: https://energy.gov/sites/prod/files/2014/03/f13/ape00a_rogers_2013_o.pdf

[2] S. Günther, S. Ulbrich, W. Hofmann, Driving cycle-based design optimization of interior permanent magnet synchronous motor drives for electric vehicle application, 2014 International Symposium on Power Electronics, Electrical Drives, Automation and Motion (SPEEDAM), 2014

[3] T. Finken, M. Hombitzer, K. Hameyer, Study and comparison of several permanent-magnet excited rotor types regarding their applicability in electric vehicles, Emobility - Electrical Power Train, 2010

[4] W. L. Soong, Design and Modelling of Axially-Laminated Interior Permanent Magnet Motor Drives for Field-Weakening Applications, Dissertation, Glasgow, 1993.

[5] E. Nipp, Permanent Magnet Motor Drives with Switched Stator Windings, Dissertation, Stockholm, 1999.

[6] K. Sakai, N. Yuzawa, Effects of Pole Changing in a Permanent Magnet Motor, 15th European Conference on Power Electronics and Applications (EPE), 2013

[7] M. Boxriker, P. Winzer, J. Kolb and M. Doppelbauer, Increasing the Operating Range of Permanent Magnet Synchronous Motors by Switching the Winding Configurations, 2nd IEEE Southern Power Electronics Conference, 2016

[8] G. Müller, K. Vogt and B. Ponick, Berechnung elektrischer Maschinen, 6th edition, WILEY-VCH Verlag GmbH \& Co. KGaA, Weinheim, 2008.

[9] M. Boxriker, J. Kolb and M. Doppelbauer, Expanding the Operating Range of Permanent Magnet Synchronous Motors by Using the Optimum Number of Phases, 18th European Conference on Power Electronics and Applications, 2016

[10] L. Parsa, On Advantages of Multi-Phase Machines, 31st Annual Conference of IEEE Industrial Electronics Society, IECON, 2005, pp.6

[11] Y. Burkhardt, A. Spagnolo, P. Lucas, M. Zavesky and P. Brockerhoff, Design and analysis of a highly integrated 9-phase drivetrain for EV applications, IEEE Transactions on Industrial Electronics, 2008, pp. 1893 - 1909

[12] A. Muetze, Bearing Currents in Inverter-Fed AC-Motors, dissertation in D 17 Darmstaedter Dissertationen,2004.

[13] B. Gagas, T. Fukushige, N. Limsuwan, C. Yu, K. Akatsu and R. Lorenz Suggested Design Space in a PMSM Parameter Plane for Variable Flux Machines,2013 International Electric Machines \& Drives Conference, 2013, pp. 549 - 556

[14] L. Hao, C. Namuduri, S. Gopalakrishnan and C. Freitas, Comparison of the Influence of PM Drive System with Voltage Adaptation or Machine Winding Reconfiguration on HEV/EV Applications, IEEE Energy Conversion Congress and Exposition (ECCE), 2015, pp. 6130 - 6138.

[15] L. Hao, C. Namuduri, S. Naik and C. Freitas, High Speed Performance of PM Machine with Reconfigurable Winding, IEEE Energy Conversion Congress and Exposition (ECCE), 2015, pp. 1840 - 1848.

[16] B. S. Umesh, M. Rao A, K. S. Kumar, Pole-Phase Modulated Multiphase Induction Motor Drive with Improved Dc Link Utilization, 42nd Annual Conference of the IEEE 2016 Industrial Electronics Society, 2016

[17] T. Bülo, Methode zur Evaluation leistungselektronischer Schaltungstopologien für die Anwendung in dezentralen Netzeinspeisern kleiner Leistung, Dissertation, Kassel University Press, Kassel, 2010 